\title{
Intelligent Shoulder Joint Home-Based Self-Rehabilitation Monitoring System
}

\author{
Jiann-I Pan ${ }^{* 1}$, Hui-Wen Chung ${ }^{1}$ and Jan-Jue Huang ${ }^{2}$ \\ ${ }^{1}$ Department of Medical Informatics, Tzu-Chi University, Hua-Lien, Taiwan \\ ${ }^{2}$ Department of Rehabilitation, Buddhist Tzu-Chi General Hospital, Hua-Lien, Taiwan \\ *jipan@mail.tcu.edu.tw
}

\begin{abstract}
Shoulder joint rehabilitation exercises are considered one of the most effective treatments for reducing shoulder pain and improving the range of motion. In addition to regular supervision from professional rehabilitation staff, participation in home-based self-practice can enhance the effectiveness of treatment. Therefore, this study proposes an intelligent monitoring system for home-based self-rehabilitation. In this system, smart phones serve as the platform for integrating an accelerometer-based sensor network for monitoring the performance of rehabilitation exercises by patients with shoulder injuries. The developed sensor network comprises 2 accelerometer sensors and the built-in smart phone accelerometer that communicate using Bluetooth protocols. The following 5 monitoring exercises were included in this study: touch ear, use fingers to climb wall both facing and sideways to the wall, clockwise and counterclockwise pendulum circles, active-assisted front and side stretches, and raises hand from back. Shoulder rehabilitation activities are recognized by the Support Vector Machine algorithms and recorded on the smart phone. These records can be used by patients as a reference of their activity. The records can also be uploaded to the hospital server to assist physicians in monitoring the effectiveness of exercises. The proposed approach is low cost and can be extended to various monitoring targets by simply installing a new Android app.
\end{abstract}

Keywords: home-based self-rehabilitation, sensor network, shoulder joint injuries, smart phone, support vector machine (SVM)

\section{Introduction}

The shoulder joint is the only joint in the human body that can be rotated $360^{\circ}$. However, the shoulder joint is easily injured by various bone and muscle damage, dislocation, and degradation caused by aging. Therefore, people of all ages are likely to experience shoulder pain and a limited range of motion. The problems of shoulder pain and limited range of motion have several causes, and are extremely complex. The differential diagnoses of shoulder injuries are traumatic and non-traumatic. Traumatic injury includes sports injuries or accidents (fractures, dislocations, or muscle tears) and repetitive movement. Non-traumatic injury includes impingement syndrome, adhesive capsulitis (frozen shoulder), joint degeneration (arthritis), and cervical nerve compression.

Upper limb rehabilitation exercise therapy can reduce spasms and pain, effectively improve shoulder joint activity, and prevent the occurrence of lymphedema. However, the effectiveness of rehabilitation often cannot be determined [1]. The main reason for the limited effectiveness of rehabilitation is that patients do not adhere to the exercise program prescribed for home-based rehabilitation. The primary goal of rehabilitation is to maintain shoulder 
mobility. Patients are liable to cease therapy if they doubt the effectiveness of the rehabilitation.

Because of the popularity of smart phone devices, related applications have been extensively developed. Establishment of the intelligent mobile phone as the calculation core, combined with the accelerometer-based motion detectors can ensure the efficacy of many home care activities [1, 2]. This study presents a wearable sensor network that integrates triaxial accelerometers and smart phone sensors to form an upper limb rehabilitation exercise monitoring system. Data of rehabilitation activity are collected using wearable sensors and built-in smart phone accelerometers, and recognized using a support vector machine (SVM) algorithm.

In this study, the following five types of exercises were monitored: 1 ) touch ear (external rotation), 2) use fingers to climb wall both facing and sideways to the wall, 3) clockwise and counterclockwise pendulum circles, 4) active-assisted front (flexion) and side (abduction) stretches, and 5) back hand raises (internal rotation) .

The remainder of this paper is organized as follows: Section 2 presents a summary of the results of related research; Section 3 introduces the architecture of the proposed home-based self-rehabilitation exercise monitoring system; Section 4 details the experiment results; and Section 5 provides the research conclusion and suggestions for future research.

\section{Related Research}

Upper limb rehabilitation requires a high repeatability and long-term continuous treatment, using a rehabilitation robot to replace the manual treatment can enable patients to continuously receive accurate guidance from a physiatrist-like [3, 4]. However, rehabilitation robots are high cost, difficult to maintain, and unsuitable for general home-based selfrehabilitation.

A typical approach for monitoring rehabilitation exercises involves using cameras to capture and recognize activities [5, 6]. However, this method has several disadvantages; for examples, rehabilitation locations are restricted, subtle movements are difficult to detect, and certain actions are performed out of the camera range.

Because of the rapid development of micro-electromechanical systems (MEMS) technology, sensors based on accelerometers and gyroscopes are widely employed for assessing and recognizing activities [1, 7-11]. Culhane et al., [8] used an accelerometer monitoring device to analyze elderly people's gait, balance, and performance of daily activities. They investigated how elderly people can safely perform rehabilitation exercises at home when alone. Patel and Hughes et al., [1] adopted an accelerometer to collect data of stroke patients who had completed actions according to the functional ability scale, and compared the calculation results with the total scale provided by medical staff. They confirmed that low-cost accelerometers can also achieve an acceptable accuracy level. Raso et al., [10] developed a monitoring system, named m-Physio, for patients with insufficient time to visit a hospital, or who resided far from the hospital, to assist them with performing rehabilitation exercises. This system combines a smart phone with network applications to form a cycle monitoring system that enables therapists to understand the condition of patients immediately. Muscillo et al., [11] proposed a real-time activity recognition method for detecting eight rehabilitation actions referenced from the Wolf Motor Function Test. Improved real-time dynamic time warping (RT-DTW) is used to recognize the actions in real time. The experiment results indicate that $60 \%$ of the actions can be identified before the end of the exercise. 
Compared with robot and vision-based approaches, micro sensors have the advantages of low cost, minimal size and weight, comparatively lower susceptibility to environmental constraints, and high portability. Thus, micro sensors can serve as the key technology of home-based self-rehabilitation exercise monitoring systems.

\section{Architecture of a Home-based Self-rehabilitation Exercise Monitoring System}

The architecture of the proposed home-based self-rehabilitation exercise monitoring system is shown in Figure 1.

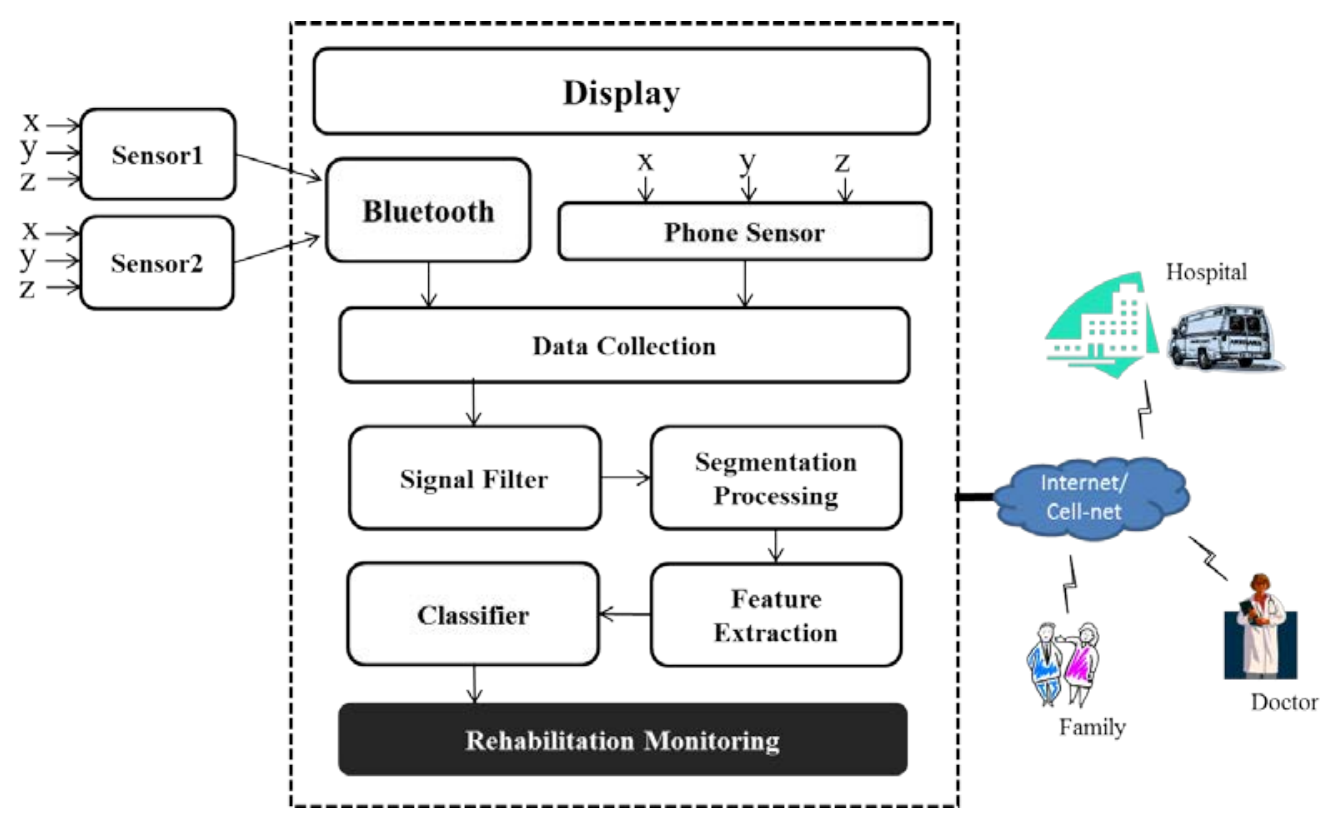

Figure 1. The Proposed System Architecture

The hardware of the proposed system comprises two accelerometer-based sensors and an Android-based smart phone. The sensors are responsible for collecting body movement data, and the smart phone serves as the main calculation platform. The primary components of the system are introduced in the following section.

\section{A. Accelerometer-based Sensor}

An accelerometer-based sensor was designed to capture body acceleration data. The sensor comprises the following components: 1) a LIS3LV02DQ triaxial acceleration sensing unit, 2) a MSP430F169 microcontroller processing unit, 3) a BTM-112 Bluetooth module wireless transmission unit, and (4) a lightweight rechargeable lithium battery as the power supply unit. The sensor dimensions are $40 \mathrm{~mm} * 28 \mathrm{~mm} * 18 \mathrm{~mm}$ (Figure 2). The sampling frequency was set as $32 \mathrm{~Hz}$.

When the system is activated, the smart phone prompts the sensor processing unit through a Bluetooth protocol to capture movement information. The triaxial acceleration sensing unit senses arm acceleration data, which it transfers to the processing unit. After receiving the acceleration data, the processing unit transfers the data to the smart phone via the Bluetooth module. The analysis and recording tasks are executed by the smart phone. 


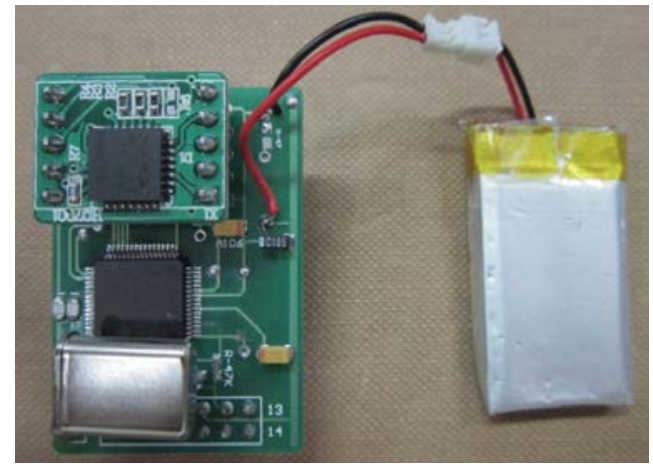

Figure 2. The Accelerometer-based Sensor

\section{B. Signal Filter}

To eliminate noise from the hardware circuit, a nonlinear median signal filter was adopted to pre-process the acceleration signal. The filtering window size was set as 7 . After applying the median filter, a low-pass (moving average) filter was used to smooth the acceleration signal. The window size was set as 30 . Figure 3 shows an example of data obtained from a wrist sensor when performing the touch ear exercise. The left image shows the original acceleration signal, and the right image shows the results of filtering using median and lowpass filters.
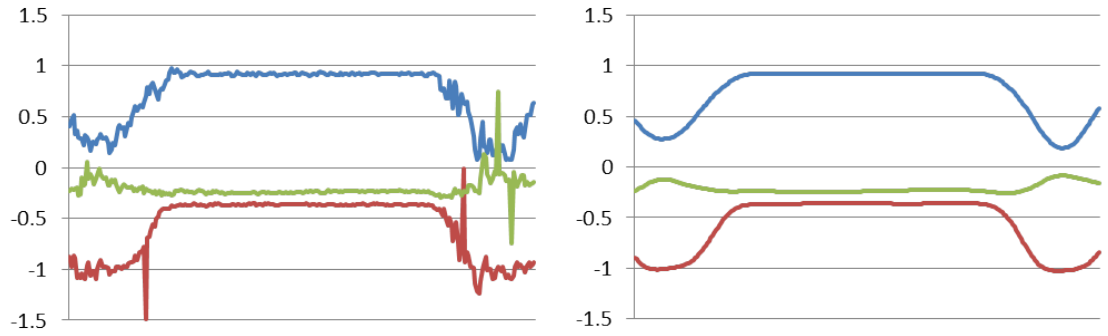

Figure 3. An Example of Signal Filtering that using Median and Low-pass Filter. The Left Hand Side Shows the Original Signal and the Right Hand Side Shows the Filtered Result

\section{Segmentation}

Accelerometer data were digitally filtered with a median filter to remove high-frequency noise and then segmented to isolate the movement actions. Patients were instructed to press the start button when beginning the periodic exercises and to press the end button after completing every exercise. Each exercise may involve one or more actions. The patients were allowed a 3-s interval to rest between actions. Segmentation was performed to distinguish the count for each action, which was conducted by the patient (Figure 4(a)). To improve the rehabilitation effects, the patients were instructed to remain stationary for at least $5 \mathrm{~s}$ when their action reached the highest range of motion (ROM) (for stopover action). This is beneficial for patient recovery.

The raw data, including that collected when stationary (static) and in a state of motion (dynamic), were segmented using a hybrid segmentation algorithm to identify separate 
activity. The segmentation algorithm comprised the following five stages: 1) calculate the first difference between stationary and in motion states (Figure 4(b)); 2) for the stationary state and initial action, perform dynamic time warping (DTW) [8] to distinguish various actions between segments (Figure 4(c)); 3) delete unnecessary signals from the beginning and end of each action; 4) using a Haar wavelet transform function [12], remove the start and end of each action (Figure 4(d)); and 5) segment detailed actions from continuous data of rehabilitation exercises (Figure 4(e)).

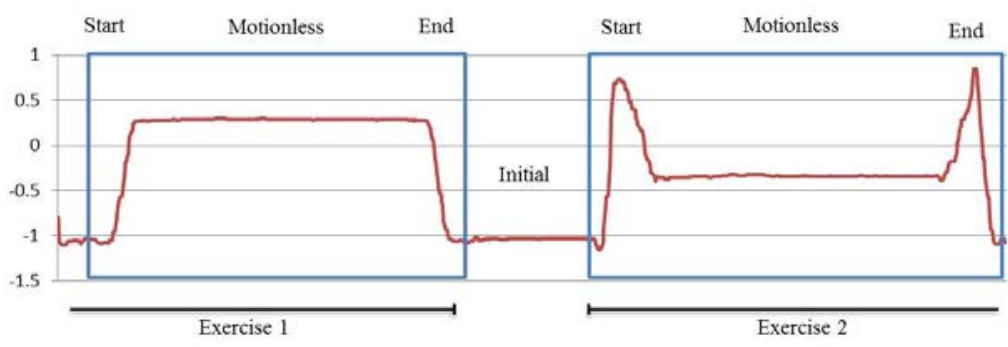

(a) Segmenting the Static and Dynamic States

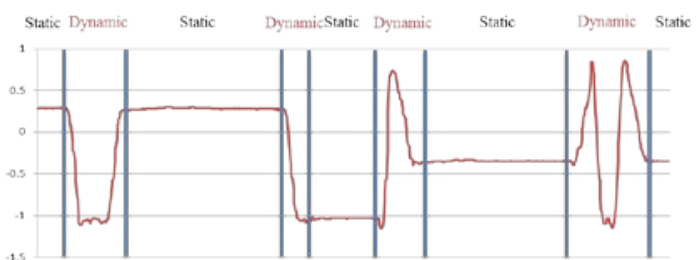

(b) Segmenting the static and dynamic states

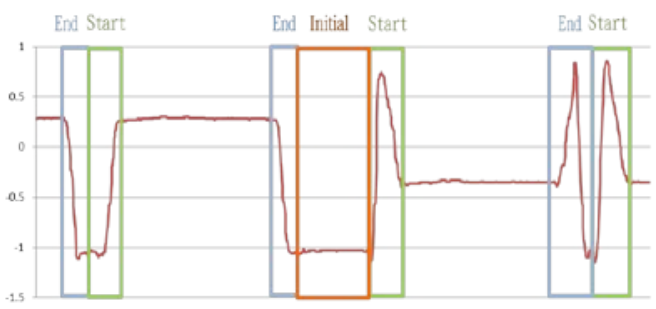

(d) Segmenting the start and end
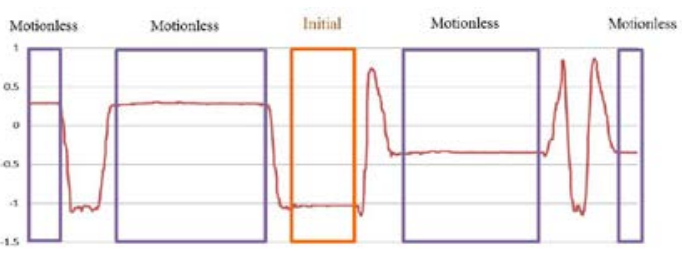

(c) Segmenting the different actions

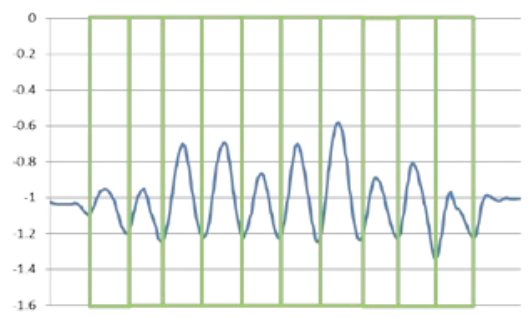

(e) Segmenting the detailed actions

Figure 4. The Five Steps in Acceleration Signal Segmentation Process

\section{Feature Extraction}

Features were extracted from the accelerometer data to capture various aspects of the activities, such as the speed, smoothness, and coordination. Specifically, the following five features were estimated:

1) Mean value of the accelerometer time series

$$
\text { Mean }=\frac{\sum_{i=1}^{n} x_{i}}{n}
$$

2) Root mean square value of the accelerometer time series 


$$
R M S=\sqrt{\frac{\sum_{i=1}^{n} x_{i}}{n}}
$$

3) Maximum value of the velocity time series

$$
\operatorname{Max}=\max \left(x_{1}, x_{2}, \ldots, x_{n}\right)
$$

4) Minimum value of the velocity time series

$$
\operatorname{Min}=\min \left(x_{1}, x_{2}, \ldots, x_{n}\right)
$$

5) Entropy of the accelerometer time series

$$
\text { Entropy }=-\sum_{i=1}^{n} p(x i) \log _{10} p\left(x_{i}\right) \text { where } p(x i)=\frac{x_{i}{ }^{2}}{\sum_{j=1}^{n} x_{j}{ }^{2}}
$$

\section{E. Classifier}

In this study, the SVM [13] algorithm was adopted as the core classification algorithm. SVM classification is widely employed for binary data classification and regression. Regarding categories, to identify the largest category edge interval (hyperplane), classification errors must be minimized. Because various rehabilitation activity data are input into the system, SVM processing follows a one-versus-all method, that is, the problem is divided into $\mathrm{N}$ classification categories (six types of exercises are included in this study). During the training phase, the collected training data are used to construct a support hyperplane. According to the identified features, data points are tagged with 1 when approaching one category; otherwise, if they approach other categories, they are tagged with 1. Thus, one piece of input data is tested using the $\mathrm{N}$ support hyperplane and subsequently allocated to the most appropriate category

\section{Results}

The sensor and smart phone device can be worn as shown in Figure 5. The smart phone was attached to the wrist for easy operation of the monitoring system.

A prototype of the intelligent rehabilitation monitoring system was produced. The experiment was conducted in a laboratory with 10 healthy and young adults and 14 patients ${ }^{1}$. The healthy young adults comprised three men and seven women aged between 20 and 25 years. The patients included five men and nine women aged between 44 and 67 years. To simulate the various situations that patients may encounter, each participant was required to perform the five rehabilitation exercises several times (Table 1). Every healthy young adult participant completed the exercises, using both their right and left shoulders, whereas the patients only performed the exercises using their injured shoulder. The data for two participants were incomplete and thus were excluded from this experiment. The captured data of the 10 healthy participants and seven randomly selected patients were analyzed using cross-validation. Data of the remaining five patients were extracted to serve as the validation data set.

\footnotetext{
1 This experiment was approved by the institutional review board of the Buddhist Tzu-Chi General Hospital, Taiwan (IRB102-58).
} 

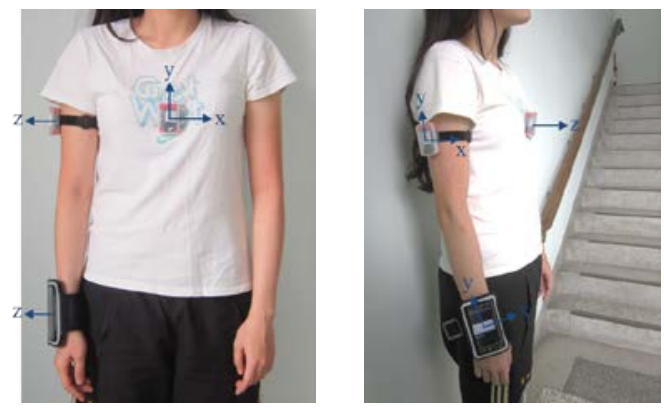

Figure 5. One Sensor and Smartphone is placed on the Affected Shoulder, and the other Sensor is placed on the Chest

Table 1. Exercise Types and Action Numbers of Training, Testing, and Validation

\begin{tabular}{|l|c|c|c|c|}
\hline \multicolumn{1}{|c|}{ Exercise types } & $\begin{array}{c}\text { Total } \\
\text { activities }\end{array}$ & Training & Testing & Validation \\
\hline Touch ear(Fig.6(a)) & 160 & 108 & 27 & 25 \\
\hline $\begin{array}{l}\text { use fingers to climb wall both } \\
\text { facing and sideways to the wall } \\
\text { (Fig.6(b)) }\end{array}$ & 320 & 216 & 54 & 50 \\
\hline $\begin{array}{l}\text { Pendulum (Fig.6(c)) } \\
\text { clockwise and counter } \\
\text { clockwise }\end{array}$ & 640 & 432 & 108 & 100 \\
\hline $\begin{array}{l}\text { Active-assisted stretch } \\
\text { (Fig.6(d)) } \\
\text { fore and side }\end{array}$ & 160 & 108 & 27 & 25 \\
\hline $\begin{array}{l}\text { raises hand from back (Fig. } \\
\text { 6(e)) }\end{array}$ & 432 & 108 & 100 \\
\hline
\end{tabular}
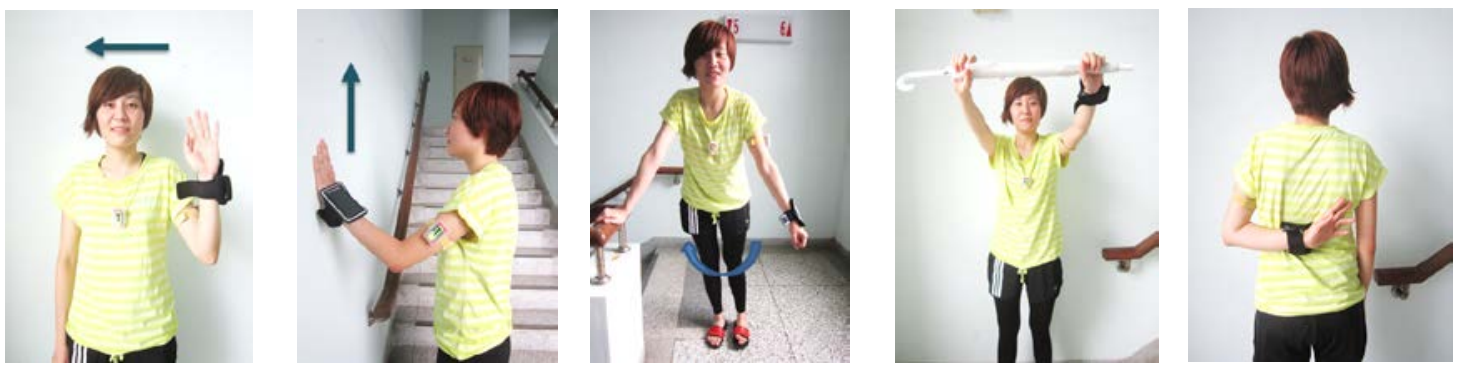

(b) Use fingers to

(a)Touch ear climb wall (facing the
(c) Pendulum counter clockwise

(d) Activeassisted stretch

(e) Raises hand from back wall)

Figure 6. The Monitored Target Activities of Rehabilitation Exercise 
A five-fold cross evaluation approach was adopted to identify the optimal classification model. Training and testing data for each exercise type were randomly divided into five subsets. The results of the proposed classification model are shown in Table 2.

Table 2. The Testing Results of Classification Model

\begin{tabular}{|c|c|c|c|c|c|c|c|c|c|}
\hline & \multirow[b]{2}{*}{$\begin{array}{l}\text { Segmenting } \\
\text { error }\end{array}$} & \multicolumn{5}{|c|}{ Classified results } & \multirow[b]{2}{*}{ Accuracy } \\
\hline & & & & $\begin{array}{c}\text { Touch } \\
\text { ear }\end{array}$ & $\begin{array}{c}\text { Raises } \\
\text { hand } \\
\text { from } \\
\text { back }\end{array}$ & $\begin{array}{c}\text { Use } \\
\text { fingers } \\
\text { to } \\
\text { climb } \\
\text { wall } \\
\end{array}$ & Pendulum & $\begin{array}{l}\text { Active- } \\
\text { assisted } \\
\text { stretch }\end{array}$ & \\
\hline \multirow{5}{*}{ Real } & Touch ear & 27 & -2 & 25 & 0 & 0 & 0 & 0 & $92.6 \%$ \\
\hline & $\begin{array}{c}\text { Raises hand } \\
\text { from back }\end{array}$ & 27 & -1 & 0 & 26 & 0 & 0 & 0 & $96.3 \%$ \\
\hline & \begin{tabular}{|c|} 
Use fingers \\
to climb wall
\end{tabular} & 54 & +1 & 0 & 0 & 55 & 0 & 0 & $98.1 \%$ \\
\hline & Pendulum & 108 & -6 & 0 & 0 & 0 & 102 & 0 & $94.4 \%$ \\
\hline & \begin{tabular}{|c|} 
Active- \\
assisted stretch
\end{tabular} & 108 & -1 & 0 & 0 & 0 & 0 & 107 & $99.1 \%$ \\
\hline
\end{tabular}

The data of five independent participants (patients) were used to further validate the effectiveness of the classification model. The overall average correctness reached $100 \%$, with a standard deviation of 0.02 (Table 3).

Table 3. Using Independent Validation Subjects to Validate the Experiment Results

\begin{tabular}{|l|l|l|l|l|l|l|}
\hline \multicolumn{5}{|c|}{ Validation Subjects } & & \\
\hline S1 & S2 & S3 & S4 & S5 & $\begin{array}{l}\text { Average } \\
\text { correctness }\end{array}$ & $\begin{array}{l}\text { Standard } \\
\text { deviation }\end{array}$ \\
\hline $96.6 \%$ & $96.6 \%$ & $97.2 \%$ & $96.9 \%$ & $97.8 \%$ & $96.85 \%$ & 0.005 \\
\hline
\end{tabular}

The experiment results indicate that errors primarily resulted from incorrect segmentation caused by the participants performing free and continuous activity. The segmenting correctness rate was $92.5 \%$ for the touching ear exercise (152/160), $98.1 \%$ for the back hand raise exercise (157/160), 97.2\% for the finger wall climb exercise (1-9/320), 95.2\% for the pendulum clockwise exercise (609/640), and $99.7 \%$ for the active-assisted stretch exercise (638/640).

\section{Conclusion and Future Research}

This study presented a low-cost expandable approach to monitoring home-based shoulder joint rehabilitation. Two accelerometer-based sensors and a built-in smart phone accelerometer captured the rehabilitation exercise data, which were then recognized by the SVM classification algorithm implemented in the smart phone. The proposed home-based self-rehabilitation exercise monitoring system provides the following three main benefits: 1) visibility: physiatrists can monitor adherence to the prescribed exercise program, that is, the actual daily rehabilitation duration, by referencing the exercise records stored on the smart phone; 2) portability: the system is not limited to a specific location, which allows 
rehabilitation exercises to be performed at any time and place; and (3) extendibility: the functions of the software installed on the smart phone can be extended or updated through the standard app update procedure, without hardware modifications.

In the future, such situations can be recognized immediately to ensure an appropriate response and avoid causing secondary damage during home-based self-rehabilitation. Furthermore, an automatic ROM measurement algorithm that serves as the basic function of the proposed rehabilitation monitoring system is set for development.

\section{Acknowledgments}

This project was supported by the National Science Council of Taiwan (Grant No. NSC 101-2218-E-320 -003).

\section{References}

[1] S. Patel, R. Hughes, T. Hester, J. Stein, M. Akay, J. G. Dy and P. Bonato, “A Novel Approach to Monitor Rehabilitation Outcomes in Stroke Survivors using Wearable Technology”, IEEE, vol. 98, no. 3, (2010) March, pp. 450-461.//conference.

[2] A. K. Bourke, J. V. O’Brien and G. M. Lyons, "Evaluation of a threshold-based tri-axial accelerometer fall detection algorithm”, Journal of Gait and Posture, vol. 26, no. 2, (2007), pp. 194-199.

[3] J. Y. Chang, "Robot-aided design for neuro-rehabilitation on strokes: assessment and therapy of upper extremity”, Doctoral dissertation, National Cheng Kung University, Department of institute of biomedical engineering, (2009).

[4] P. S. Lum, C. G. Burgar, P. C. Shor, M. Majmundar and M. Vander Loos, "Robot-assisted movement training compared with conventional therapy techniques for the rehabilitation of upper-limb motor function after stroke”, Archives of Physical Medicine and Rehabilitation, vol. 83, (2002), pp. 952-959.

[5] J. Appenrodt, A. Al-Hamadi and B. Michaelis, "Data Gathering for Gesture Recognition Systems Based on Single Color-, Stereo Color- and Thermal Cameras”, International Journal of Signal Processing, Image Processing and Pattern Recognition,vol. 3, no. 1, (2009), pp. 37-50.

[6] H. Ali, J. Dargham, C. Ali and E. G. Moung, "Gait Recognition using Gait Energy Image”, International Journal of Signal Processing, Image Processing and Pattern Recognition, vol. 4, no. 3, (2011), pp. 141-152.

[7] J. Wang, Z. Zhang, Y. Zheng, M. Wu and J. Kim, “A Novel Fall Activity Recognition Method for Wireless Sensor Networks”, International Journal of u- and e- Service, Science and Technology, vol. 5, no. 4, (2012), pp. 1-14.

[8] K. M. Culhane, M. O’Connor, D. Lyons and G. M. Lyons, “Accelerometers in rehabilitation medicine for older adults”, Age and Ageing, vol. 34, no. 6, (2005) October, pp. 556-560.

[9] J. P. Giuffrida, A. Lerner, R. Steiner and J. Daly, "Upper-extremity stroke therapy task discrimination using motion sensors and electromyography”, IEEE Transactions Neural Systems and Rehabilitation Engineering, vol. 16, no. 1, (2008) February, pp. 82-90.

[10] I. Raso, R. Hervás and J. Bravo, "m-Physio: ersonalized accelerometer-based physical rehabilitation platform”, The Fourth International Conference on Mobile Ubiquitous Computing, Systems, Services and Technologies, IARIA, (2010), pp. 416-421.

[11] R. Muscillo, M. Schmid, S. Conforto and T. D’Alessio, "Early recognition of upper limb motor tasks through accelerometers: real-time implementation of a DTW-based algorithm”, Computers in Biology and Medicine, vol. 41, no. 3, (2011), pp. 164-172.

[12] A. Haar, "Zur theorie der orthogonalen funktionen systeme”, Mathematische Annalen, vol. 69, (1910), pp. 331-371.

[13] C. C. Chang and C. J. Lin, “LIBSVM: A Library for Support Vector Machines”, Journal of ACM Transactions on Intelligent Systems and Technology, vol. 2, no. 3, (2011), Article 27, pp. 27. 


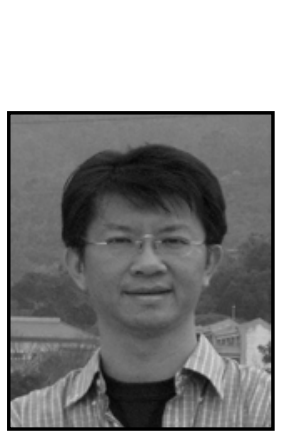

\section{Authors}

Jiann-I Pan received a BEng degree in Electronic Engineering from National Taiwan University of Science and Technology, Taiwan, in 1991. Then, in 1995 and 1999, Pan earned MSc and PhD degrees in Computer Science and Information Engineering from National Central University, Taiwan. He currently serves as an assistant professor at Tzu Chi University, Taiwan. His research interests include software engineering in the development of medical information systems, intelligent human activity monitoring systems, and sensor networks.

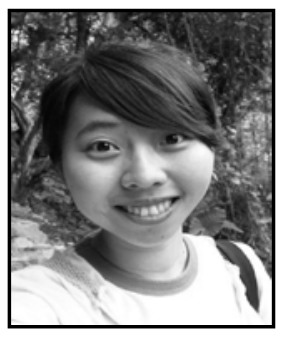

Hui-Wen Chung received a BSc degree in Medical Informatics from Tzu Chi University, Taiwan, in 2010. She is currently working toward an MSc degree at the Department of Medical Informatics, Tzu Chi University, Taiwan. Her research interests include human activity recognition and data mining.

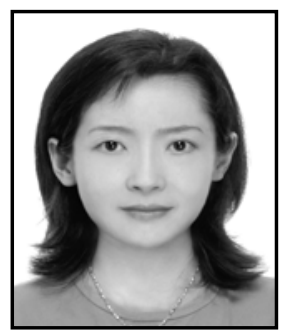

Jan-Jue Huang received a BSc degree in Physical Therapy from Kaohsiung Medical University, Taiwan, in 1992. She subsequently earned an MSc degree in Physical Therapy from Massachusetts General Hospital Institute of Health Professions, Boston, USA, in 1999. She currently serves as a physical therapist at Tzu Chi General Hospital, Taiwan. 